\title{
Umbilical cord-derived mesenchymal stem cells promote proliferation and migration in MCF-7 and MDA-MB-231 breast cancer cells through activation of the ERK pathway
}

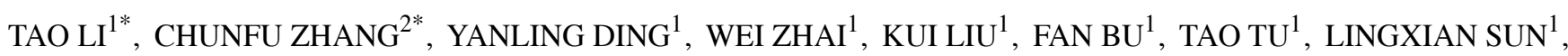 \\ WEI ZHU ${ }^{1}$, FANGFANG ZHOU ${ }^{1}$, WENKAI QI ${ }^{1}$, JIABO HU ${ }^{1}$, HUABIAO CHEN ${ }^{1,3}$ and XIAOCHUN SUN ${ }^{1}$ \\ ${ }^{1}$ School of Medicine, Jiangsu University, Jiangsu Key Laboratory of Clinical Laboratory Medicine, Zhenjiang, \\ Jiangsu 212013; ${ }^{2}$ The Second People's Hospital of Kunshan, Kunshan, Jiangsu 215300, P.R. China; ${ }^{3}$ Vaccine and \\ Immunotherapy Center, Massachusetts General Hospital and Harvard Medical School, Boston, MA 02114, USA
}

Received April 22, 2015; Accepted June 3, 2015

DOI: 10.3892/or.2015.4109

\begin{abstract}
Mesenchymal stem cells (MSCs) are known to migrate to tumor tissues and to play an important role in cancer progression. However, the effects of MSCs on tumor progression remain controversial. The purpose of the present study was to detect the effects of human umbilical cord-derived MSCs (hUC-MSCs) on the human breast cancer cell lines MDA-MB-231 and MCF-7 in vitro and the underlying mechanisms. MSCs were isolated and identified from umbilical cord tissues. MDA-MB-231 and MCF-7 cells were treated with conditioned medium (CM) from 10 and $20 \%$ umbilical cord MSCs (UC-MSCs), and the resulting changes in proliferation and migration were investigated. The 3-(4,5-dimethyl-2-thiazolyl)-2,5-diphenyl-2-H-tetrazolium bromide (MTT) and plate clone formation assays were used to assess the effect on proliferation, and the effects of CM on MDA-MB-231 and MCF-7 migration were assessed through scratch wound and Transwell migration assays. The expression of cell proliferation- and metastasis-related genes and proteins and activation of the ERK signaling pathway were analyzed by RT-PCR and western blot assays. UC-MSCs are characteristically similar to bone marrow MSCs (BM-MSCs) and exhibit multipotential differentiation capability (i.e., osteoblasts and adipocytes). The MTT, plate clone formation, scratch wound and Transwell migration assay results revealed that 10 and $20 \% \mathrm{CM}$ promoted the prolif-
\end{abstract}

Correspondence to: Dr Xiaochun Sun or Dr Huabiao Chen, School of Medicine, Jiangsu University, Jiangsu Key Laboratory of Clinical Laboratory Medicine, 301 Xuefu Road, Zhenjiang, Jiangsu 212013, P.R. China

E-mail: xiaochun@ujs.edu.cn

E-mail: huabiao.chen@mgh.harvard.edu

${ }^{*}$ Contributed equally

Key words: umbilical cord mesenchymal stem cells, proliferation, migration, ERK, MCF-7, MDA-MB-231 eration and migration to higher levels than those observed in the control group. Our findings showed that UC-MSC-CM inhibited E-cadherin expression, increased the expression of $\mathrm{N}$-cadherin and proliferating cell nuclear antigen (PCNA) and enhanced the expression of ZEB1, a transcription factor involved in epithelial-to-mesenchymal transition (EMT), through activation of the ERK pathway. U0126, an inhibitor of ERK, reversed the effects of UC-MSC-CM on breast cancer cell proliferation and migration. We conclude that UC-MSCs promote the proliferation and migration of breast cancer cell lines via activation of the ERK pathway.

\section{Introduction}

Breast cancer is the most common malignancy and is the leading cause of cancer-related death in females worldwide $(1,2)$. At present, the main clinical therapy strategies include chemotherapy, surgery and radiotherapy, but all of these have side effects $(3,4)$. Mesenchymal stem cells (MSCs) are known to migrate to tumors, and it is possible to exploit the behavior of MSCs as a tumor-targeting method for cell-based cancer therapy. The effects of MSCs on tumor progression remain controversial, and in particular, it is not clear whether the clinical application of MSCs leads to unforeseen and unwanted side effects.

Tumor development has been recognized as the result of the interaction between tumor cells and their surrounding supporting tissues (5). The mutual interactions of tumor cells and stromal cells through direct contact to various cytokines and chemokines in a paracrine manner are thought to modulate tumor progression (6-8). Several studies indicate that MSCs promote tumor proliferation and metastasis $(9,10)$, whereas other studies suggest that MSCs display intrinsic anticancer activities (11-13). This discrepancy requires further investigation.

The bone marrow is the main source of MSCs, but their collection from the bone marrow is extremely difficult. The proliferative and multilineage differentiation capacities of bone marrow-derived MSCs (BM-MSCs) decreases with aging (14). However, umbilical cord collection is convenient 
and is not associated with any ethical or legal issue (15). Many studies have confirmed that the proliferative and differentiation abilities of umbilical cord MSCs (UC-MSCs) are greater than those of BM-MSCs (16). Therefore, UC-MSCs are considered a promising source of stem cells for cancer therapy.

UC-MSCs have been found to target many primary solid tumors and their metastases $(17,18)$. UC-MSCs secrete interferon-b (IFN-b), which was found to reduce the growth of human MDA-MB-231 breast carcinoma cells by inducing apoptosis (19). It was recently shown that the intratumoral injection of rat umbilical cord matrix stem cells (rUC-MSCs) caused regression of rat mammary carcinomas (20). Human umbilical cord Wharton's jelly stem cells (hWJSCs) have been shown to have anti-inflammatory potential by reducing the expression of inflammatory mediators (21). Taken together, the results indicate that UC-MSCs exhibit an anticancer effect. However, it remains unclear whether UC-MSCs are safe in cancer clinical therapy.

The MDA-MB-231 cell line is a triple-negative breast cancer cell line (22) that exhibits stronger drug resistance and a tendency to manifest recurrence and metastasis. The MCF-7 cell line is an estrogen receptor-positive, hormone-dependent breast cancer cell line. In the present study, we sought to ascertain whether UC-MSCs have the capability to affect the migratory potential of MCF-7 cells, which have very low metastatic potential (23), and whether UC-MSCs exert differential effects in MDA-MB-231 and MCF-7 cells. The molecular mechanism of UC-MSCs on cancer cells remains unclear. Thus, a better understanding of the molecules or mechanism that regulates the proliferative and migratory behaviors of breast cancer cells is essential to the development of novel effective therapies. Thus, the present study focused on the molecular mechanism underlying these effects.

\section{Materials and methods}

Cell culture. The cells were cultured in Dulbecco's modified Eagle's medium with low glucose (L-DMEM) supplemented with $10 \%$ fetal bovine serum (FBS; Gibco, Carlsbad, CA, USA), $100 \mathrm{U} / \mathrm{ml}$ penicillin and streptomycin under mycoplasma-free conditions at $37^{\circ} \mathrm{C}$ in $5 \% \mathrm{CO}_{2}$. The human breast cancer cell lines MCF-7 and MDA-MB-231 were a gift from Dr W. Zhu (Department of Medicine, Jiangsu University, China).

Isolation and culture of human umbilical cord MSCs ( $h U C-M S C s)$. Fresh umbilical cords were collected from informed, consenting mothers at the First People's Hospital of Zhenjiang (China) and rapidly processed. Moreover, hUC-MSCs were isolated within the optimal processing period of $6 \mathrm{~h}$. The cords were rinsed twice with phosphate-buffered saline (PBS) supplemented with penicillin and streptomycin to remove any blood and cord vessels. The washed cords were subsequently cut into $1 \mathrm{~mm}^{3}$ pieces, floated in L-DMEM containing $10 \% \mathrm{FBS}$, penicillin and streptomycin, and incubated at $37^{\circ} \mathrm{C}$ with $5 \% \mathrm{CO}_{2}$. The medium was replaced every 3 days after the initial culture. When well-developed colonies of fibroblastlike cells appeared after 10 days, the cultures were trypsinized and passaged into a new flask for further expansion, and the medium was changed every 3 days. The experimental protocol was approved by the Jiangsu University Ethics Committee.
Flow cytometry. After the third passage, the cells were trypsinized (0.25\% trypsin EDTA), washed twice with PBS and stained on ice with monoclonal antibodies against FITC-CD34, HLA-DR, PE-CD29, CD44 and CD90 (Becton-Dickinson, San Jose, CA, USA). PE-IgG1 and FITC-IgG1 were used as isotype controls. The stained cells were analyzed by flow cytometry (FACSCalibur; Becton-Dickinson).

Osteogenic and adipogenic differentiation in vitro. The differentiation of UC-MSCs was assessed in the third passage. The cells were cultured in medium that contained either osteogenic reagents $[0.1 \mu \mathrm{M}$ dexamethasone, $10 \mathrm{mM} \beta$-glycerophosphate, $50 \mathrm{mg} / \mathrm{l}$ ascorbic acid and $4 \mu \mathrm{g} / \mathrm{ml}$ basic fibroblast growth factor (bFGF)] (all from Sigma-Aldrich, St. Louis, MO, USA) for 2 weeks or adipogenic reagents $(1 \mu \mathrm{M}$ dexamethasone, $0.5 \mu \mathrm{M} 3$-isobutyl-1-methylxanthine, $5 \mathrm{ng} / \mathrm{ml}$ insulin, $60 \mu \mathrm{M}$ indomethacin and $100 \mu \mathrm{M}$ hydrocortisone) (all from Cyagen, Guangzhou, China) for 3 weeks. Two or three weeks later, the degree of osteogenic differentiation was assessed by Alizarin Red staining, and the intracellular lipid accumulation was visualized by Oil Red O staining.

Generation of conditioned media. UC-MSCs were plated to $70 \%$ confluency in 35-mm plates with $10 \%$ FBS L-DMEM and allowed to adhere overnight at $37^{\circ} \mathrm{C}$ in $5 \% \mathrm{CO}_{2}$. The following day, the media was removed, and the cells were washed twice with PBS. The cells were then re-incubated with non-serum culture media. After $12 \mathrm{~h}$, the conditioned medium (CM) was collected and passed through a $0.45-\mu \mathrm{m}$ filter (Sigma-Aldrich). $\mathrm{CM}$ aliquots were frozen at $-20^{\circ} \mathrm{C}$ until required (not exceeding 2 weeks). To prepare different concentrations of UC-MSC-CM (10 and 20\%), 100\% UC-MSC-CM was diluted accordingly in freshly prepared L-DMEM with $10 \%$ FBS.

MTT and plate colony formation assays. The cells were plated at a density of $2.5 \times 10^{3}$ cells/well in a 96 -well plate in $180 \mu \mathrm{l}$ of L-DMEM and allowed to attach overnight. The cells were then treated with 0,10 and $20 \% \mathrm{CM}$ for $48 \mathrm{~h}$. MTT $(20 \mu \mathrm{l})$ was added to each well for the last $4 \mathrm{~h}$. Once the reaction was terminated, the solution was discarded, and $150 \mu \mathrm{l}$ of dimethyl sulfoxide was added to each well. The 96-well plate was shaken to ensure complete solubilization of the purple formazan crystals. The absorbance at $490 \mathrm{~nm}$ was measured using an enzyme-linked immunosorbent assay reader. For the colony formation assay, the cells were plated at a density of 200 cells/plate in a 6-well plate. After culturing for 10 days, the colony units were fixed with methanol and stained with crystal violet for $30 \mathrm{~min}$ before washing with water and air-drying. The clones with $>150$ cells were counted with an optical microscope, and the clone formation rate was calculated using the following formula: Plate clone formation efficiency $=$ (number of clones/number of cells inoculated) $x 100 \%$. All of the experiments were repeated 3 times, and the average values are reported.

Scratch wound assay. The cells were grown to confluence and then scratched with a $0.2-\mathrm{ml}$ pipette tip. The resulting debris was removed by gentle washing with medium. The cells were subsequently placed in an incubator. The cells were maintained in the presence of 0,10 and $20 \% \mathrm{CM}$ for $24 \mathrm{~h}$, respectively. Images of the closing wound were acquired with 
an inverted microscope and analyzed using Image software (National Institutes of Health, Bethesda, MD, USA).

Transwell migration assay. First, 0, 10 or 20\% CM was added to the bottom chambers. Then, $5 \times 10^{4} \mathrm{MCF}-7$ cells and $2.5 \times 10^{4}$ MDA-MB-231 cells were plated in $100 \mu$ l of non-serum L-DMEM and added to the top of the chambers (Corning, Lowell, MA, USA), and the plates were then incubated for $12 \mathrm{~h}$ at $37^{\circ} \mathrm{C}$. The cells on the top part of the filter were removed by scrubbing twice with a cotton swab. The migrating cells were fixed in formaldehyde and stained with crystal violet. Four low-power fields (magnification, x200) were randomly selected from each chamber to observe the cells and count the stained migrated cells. Each experimental group was assessed in triplicate.

Reverse transcriptase-polymerase chain reaction (RT-PCR). MCF-7 and MDA-MB-231 cells were treated with CM $(0,10$ or $20 \%)$ for $48 \mathrm{~h}$. The total RNA was isolated with the TRIzol reagent (Invitrogen), and $1 \mu \mathrm{g}$ of RNA was processed for cDNA synthesis with Superscript II reverse transcriptase using Oligo-dT primer (Toyobo, Japan). PCR was performed using $1 \mu \mathrm{g}$ of cDNA sample with $0.3 \mathrm{U}$ of Taq polymerase (Cinnagen, Iran), $200 \mu \mathrm{M}$ dNTPs, $10 \mathrm{pM}$ of each primer, reaction buffer, and $\mathrm{MgCl}_{2}$ (Takara, Japan) in a $25-\mu \mathrm{l}$ volume. PCR amplification was performed for 35 cycles using an ABI 2720 Thermal Cycler (Applied Biosystems). The cycling conditions were $94^{\circ} \mathrm{C}$ for $30 \mathrm{sec}, 60^{\circ} \mathrm{C}$ (primer) for $30 \mathrm{sec}$, and $72^{\circ} \mathrm{C}$ for $30 \mathrm{sec}$, and a final extension at $72^{\circ} \mathrm{C}$ for $10 \mathrm{~min}$ was performed. The PCR products were separated on a $1.5 \%$ agarose gel, stained with ethidium bromide, and visualized under UV light. The specific primers for PCR were designed as follows: $\beta$-actin sense, 5'-CACGAAACTACCTTCAACTC-3' and antisense, 5'-CATACTCCTGCTTGCTGATC-3'; E-cadherin sense, 5'-CGCATTGCCACATACACTCT-3' and antisense, 5'-TTGG CTGAGGATGGTGTAAG-3'; and N-cadherin sense, 5'-AGT CAACTGCAACCGTGTCT-3' and antisense, 5'-AGCGTTCC TGTTCCACTCAT-3'.

Western blot assay. MCF-7 and MDA-MB-231 cells were treated with CM (0, 10 and 20\%) for $48 \mathrm{~h}$. The total cellular protein was extracted using RIPA lysis buffer. Samples containing $100 \mu \mathrm{g}$ of protein were separated on $10 \%$ SDS-PAGE gels (Beyotime, Shanghai, China) and transferred electrophoretically to a PVDF membrane (Millipore Corp., Billerica, MA, USA), and the membrane was then blocked with $5 \%$ (w/v) skim milk in TBST $(20 \mathrm{mM}$ Tris- $\mathrm{HCl}, 0.15 \mathrm{M}$ $\mathrm{NaCl}$, and $0.05 \%$ Tween-20) for $1 \mathrm{~h}$ at room temperature. The membranes were then incubated with primary antibodies at $4^{\circ} \mathrm{C}$ overnight, washed in TBST and incubated for $1 \mathrm{~h}$ with a goat anti-rabbit secondary antibody. The reactions were visualized using an ECL detection system (Amersham Pharmacia Biotech, Little Chalfont, UK). The western blot data are representatives from 3 independent experiments. The intensities of the bands obtained from the western blot assays were quantified using the Gel Image analysis software (Lane 1D, Beijing, China). The following primary antibodies were used: p-ERK and T-ERK (1:1,000; Santa Cruz Biotechnology, Santa Cruz, CA, USA), PCNA (1:1,000; Bioworld, Minneapolis, MN, USA), E-cadherin, N-cadherin and ZEB1 (1:1,000; Cell
Signaling Technology, Beverly, MA, USA) and GAPDH and the secondary antibody (1:2,000; Kangcheng Bio-Engineering, Shanghai, China).

Statistical analysis. Differences between more than two groups were analyzed by one-way ANOVA with the NewmanKeuls multiple comparison test using the GraphPad Prism V5.0 software program (GraphPad, San Diego, CA, USA). The results are expressed as the mean $\pm \mathrm{SD}$ of 3 different replicates from individual assays. $\mathrm{p}<0.05, \mathrm{p}<0.01$ and $\mathrm{p}<0.001$ were considered to indicate statistically significant differences.

\section{Results}

Morphology and differentiation potential and surface antigens of UC-MSCs. After 7 to 10 days of initial culture, the long spindle-shaped fibroblastic cells began to form colonies and became confluent (Fig. 1A). A multilineage differentiation potential is the functional standard for verifying the identity of MSCs. The differentiation of UC-MSCs was apparent after 2 or 3 weeks of induction under specific media. At the end of the second or third week, the UC-MSCs were capable of differentiating into osteocytes and adipocytes, as shown by positive staining of Oil Red O (Fig. 1B) and Alizarin Red (Fig. 1C). The surface antigens of MSCs were positive for CD29, CD44, and CD90 but negative for CD34 and HLA-DR (Fig. 1D).

UC-MSCs enhance the proliferation of MCF-7 and $M D A-M B-231$ cells. We hypothesized that various soluble factors secreted by stem cells are capable of affecting cancer cell growth; thus, we further investigated the effects of CM derived from UC-MSCs on breast cancer cells. The MTT assay of MCF-7 and MDA-MB-231 cells cultured in UC-MSC-CM $(0,10$ and $20 \%)$ revealed proliferation rates of $0.342 \pm 0.015$, $0.557 \pm 0.066$ and $0.534 \pm 0.047$ for MCF-7 cells and $0.143 \pm 0.017$, $0.275 \pm 0.046$ and $0.299 \pm 0.060$ for MDA-MB-231 cells, respectively (Fig. 2A). The increases in the proliferation rates of MCF-7 and MDA-MB-231 cells with 10 and 20\% CM were statistically significant compared with the rates observed in the control groups $(\mathrm{p}<0.05)$. After 10 days of culture, the plate clone formation rates obtained for the control group and the 10 and $20 \% \mathrm{CM}$ groups were $0.040 \pm 0.013,0.088 \pm 0.013$ and $0.173 \pm 0.025$ for MCF-7 cells and $0.080 \pm 0.010,0.157 \pm 0.018$, and $0.128 \pm 0.013$ for MDA-MB-231 cells, respectively, and these differences were statistically significant ( $<<0.05$, Fig. $2 B$ and $\mathrm{C}$ ). Our western blot results showed that treatment with $\mathrm{CM}$ increased the PCNA protein levels in breast cancer cells (Fig. 2D). The data also revealed that the PCNA expression level in MCF-7 and MDA-MB-231 cells treated with 10 and $20 \% \mathrm{CM}$ presented significant differences compared with the control groups (Fig. 2E, p<0.05).

$U C-M S C$ s promote the migration of $M C F-7$ and $M D A-M B-231$ cells. In this study, we sought to determine whether UC-MSCs affect the migratory potential of the normally non-metastatic MCF-7 cell line and the high-metastatic MDA-MB-231 cell line. In the Transwell migration assay, the mean numbers of migrated MCF-7 cells in the lower fields after $12 \mathrm{~h}$ were $46.50 \pm 12.40,77.75 \pm 6.02$ and $91.00 \pm 8.52$, whereas the 
A

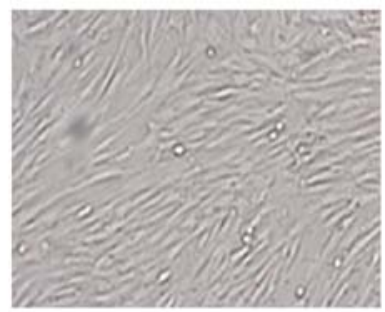

hUC-MSCs
B

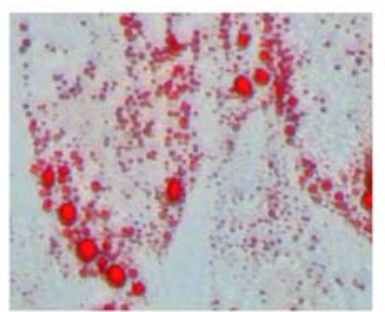

Adipogenic group
C

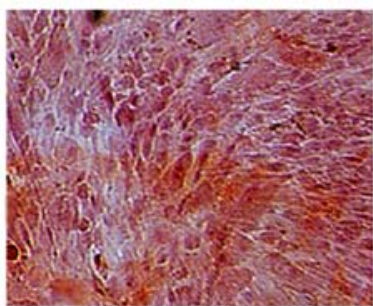

Osteogenic group
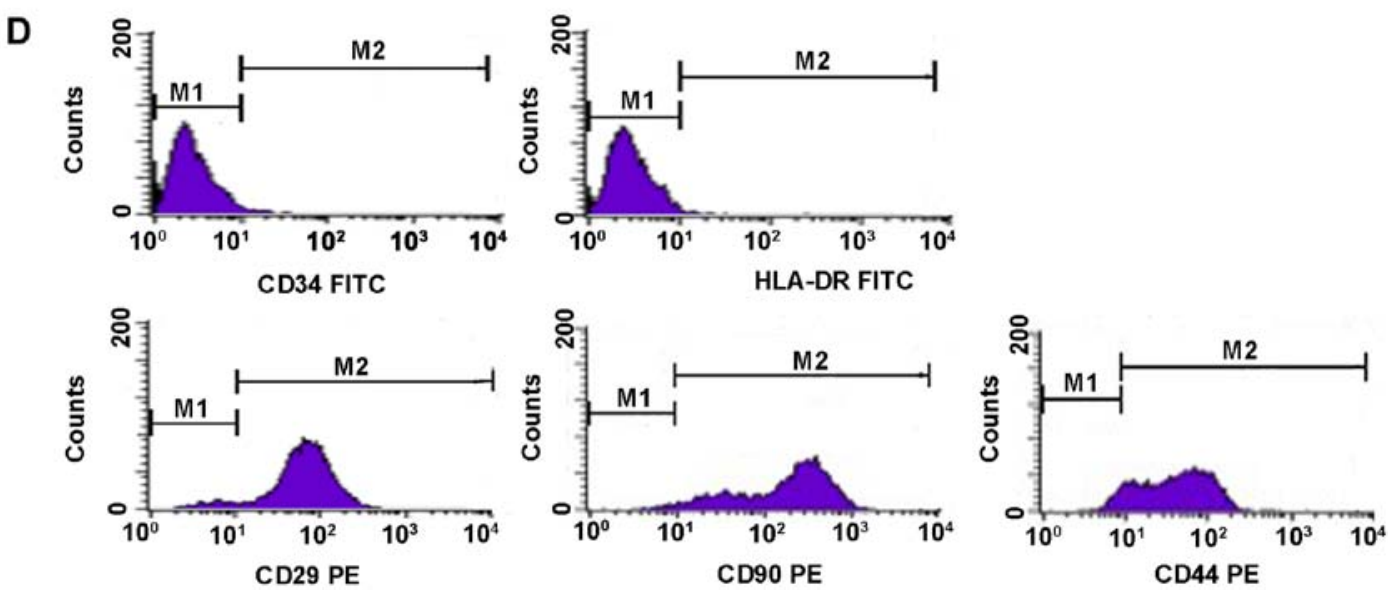

Figure 1. Morphology and differentiation potential and surface antigens of UC-MSCs. (A) UC-MSCs were long, spindle-shaped and fibroblastic in appearance after 10 days of primary culture. (B) Detection of Oil Red O staining after 3 weeks in adipogenic medium. Some of the cells contained numerous Oil Red O-positive lipid droplets. (C) Alizarin Red staining of cell cultures grown for 2 weeks in osteogenic medium. Some of the MSCs became Alizarin Redpositive. (A-C) Magnification, x200. (D) UC-MSCs are positive for CD29, CD44 and CD90 but negative for CD34 and HLA-DR. UC-MSCs, umbilical cord mesenchymal stem cells.

A

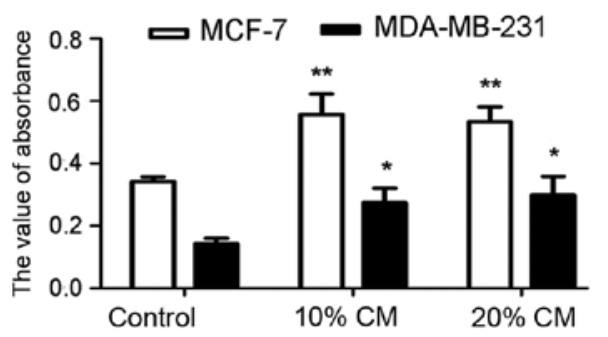

B
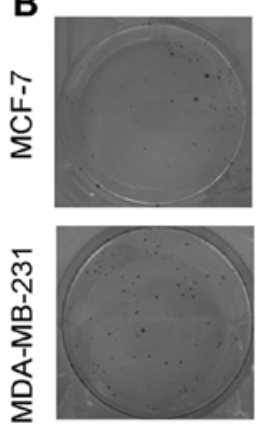

Control
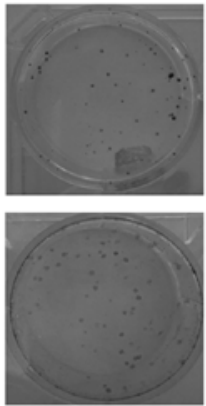

$10 \% \mathrm{CM}$
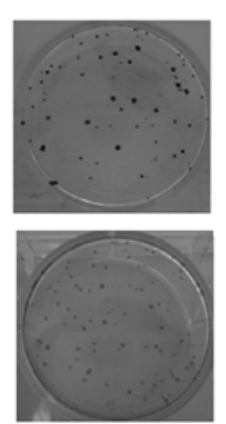

$20 \% \mathrm{CM}$

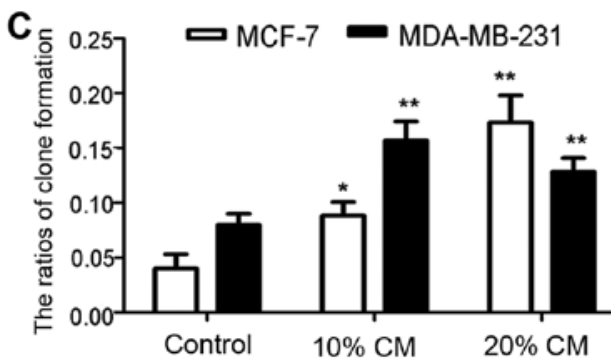

D

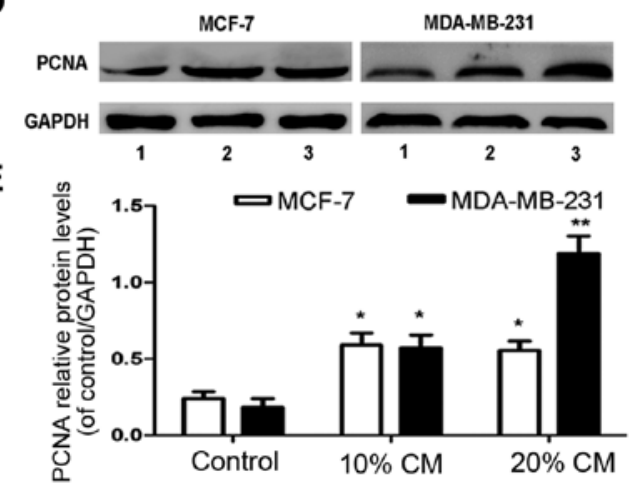

Figure 2. UC-MSCs enhance the proliferation of MCF-7 and MDA-MB-231 cells. (A) MTT assay results. (B) Clone formation images of the MCF-7 and MDA-MB-231 cells treated or untreated with UC-MSC-CM for 10 days. (C) Clone formation efficiency of the MCF-7 and MDA-MB-231 cells. (D) Western blot analysis of PCNA protein levels in the MCF-7 and MDA-MB-231 cells. The GAPDH protein was used as a control to ensure equal loading. (E) Three independent experiments were performed to determine the PCNA protein levels, and the results are presented as the means \pm SD. ${ }^{*}<<0.05$ and ${ }^{* *} \mathrm{p}<0.01 \mathrm{com}-$ pared with the control group. Lane 1, control; lane 2, 10\% CM; lane 3, 20\% CM. CM, conditioned medium; UC-MSCs, umbilical cord mesenchymal stem cells.

mean numbers of MDA-MB-231 cells were $76.50 \pm 5.97$, $112.50 \pm 10.28$, and $140.50 \pm 5.79$, respectively. There were significant differences between the control group and the CM groups ( $p<0.05$, Fig. 3A and B). Scratch wounds were 
A
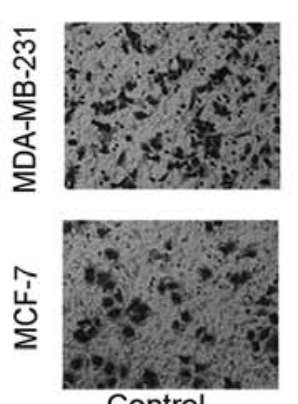

Control
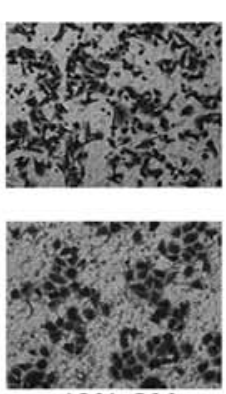

$10 \% \mathrm{CM}$
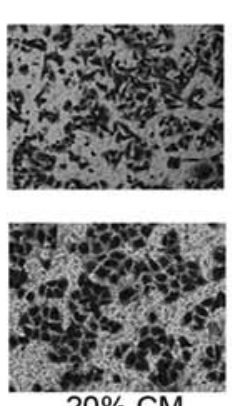

$20 \%$ CM

B

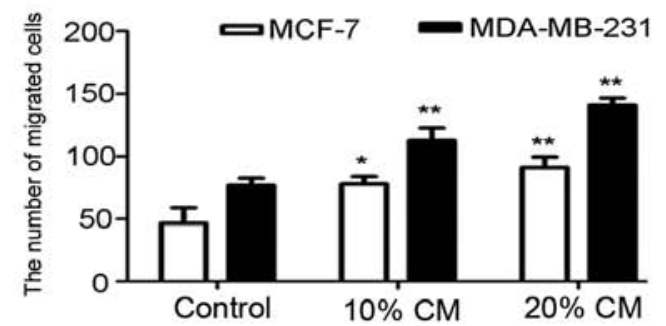

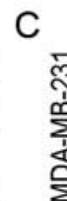
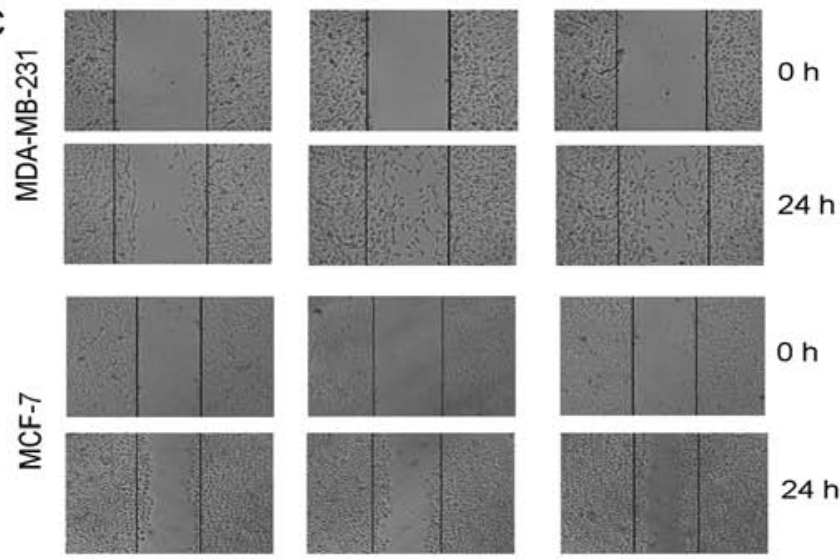

D

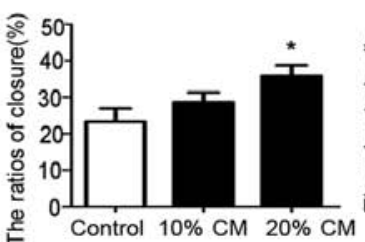

MCF-7

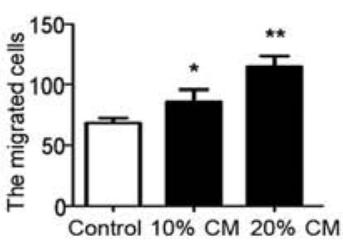

MDA-MB-231

Figure 3. UC-MSCs promote the migration of MCF-7 and MDA-MB-231 cells. (A) Results of the Transwell migration assay of MCF-7 and MDA-MB-231 cells. (B) Number of migrated cells in the different groups. There were significant differences between the 10 and $20 \%$ CM groups and the control groups in terms of the number of migrated cells. Magnification, x200. (C) Wound-healing assay results. Example images of wound closure in the control, 10 and $20 \%$ CM groups. (D) Wound closure ratios in the different groups. There were significant differences between the 10 and $20 \% \mathrm{CM}$ groups and the control groups. ${ }^{*} \mathrm{p}<0.05$ and $^{* *} \mathrm{p}<0.01$ compared with the control group. Magnification, x100. CM, conditioned medium; UC-MSCs, umbilical cord mesenchymal stem cells.
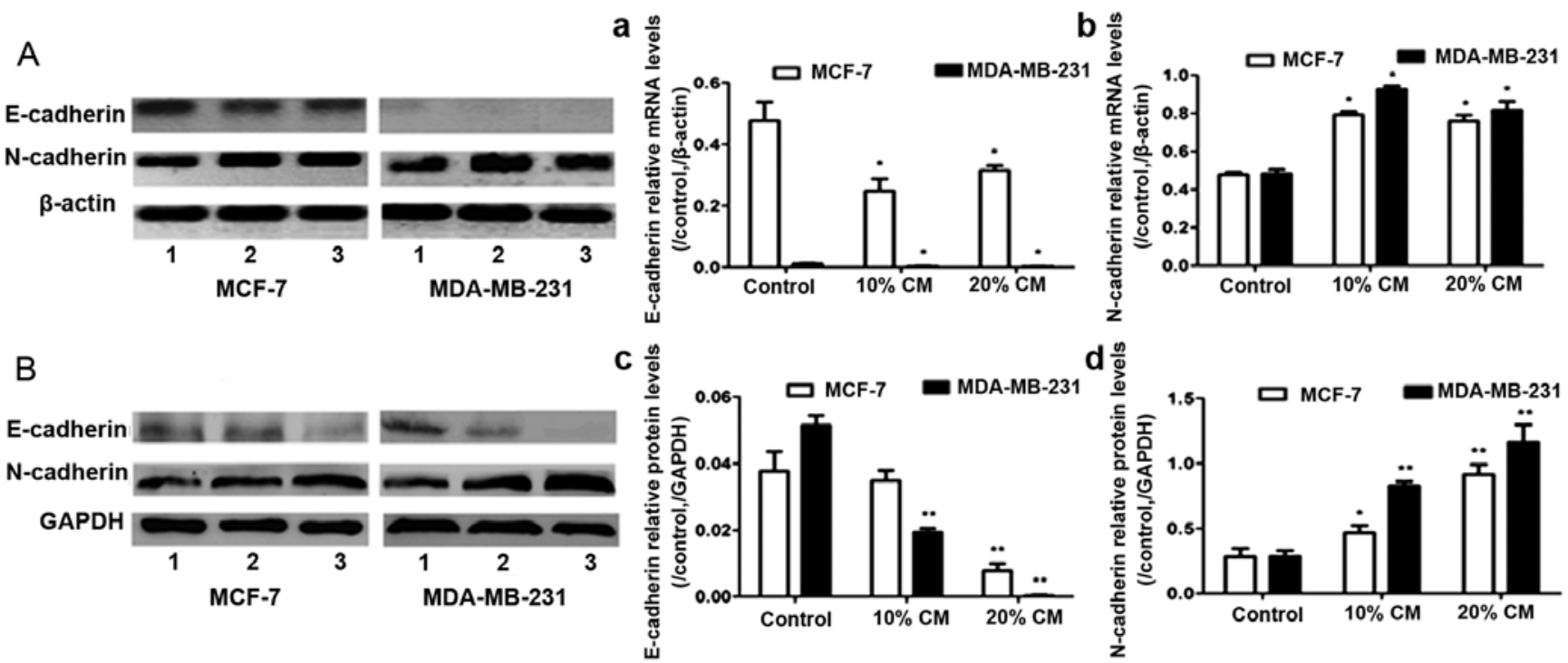

Figure 4. E-cadherin and N-cadherin expression. (A) The E-cadherin and N-cadherin mRNA levels were analyzed by RT-PCR, and $\beta$-actin mRNA was used as a control to ensure equal loading. Three independent experiments for measuring the (a) E-cadherin and (b) N-cadherin mRNA levels. (B) The E-cadherin and N-cadherin protein levels in MCF-7 and MDA-MB-231 were analyzed by western blotting. GAPDH protein was used as a control to ensure equal loading. Three independent experiments for measuring the (c) E-cadherin and (d) N-cadherin protein levels. " $\mathrm{p}<0.05$ and ${ }^{* *} \mathrm{p}<0.01$ compared with the control group. Lane 1, control; lane 2, 10\% CM; lane 3, 20\% CM. CM, conditioned medium.

inflicted in cells pretreated with or without UC-MSC-CM for $24 \mathrm{~h}$, whereas differences were observed between the groups after $24 \mathrm{~h}$ of treatment (Fig. 3C). The wound closure ratios for MCF-7 cells were $23.18 \pm 3.73,28.65 \pm 2.61$ and $35.83 \pm 2.88 \%$ in the control, 10 and $20 \% \mathrm{CM}$ groups, respectively. The difference obtained with $10 \% \mathrm{CM}$ was not significant, but that obtained with $20 \% \mathrm{CM}$ was statistically significant $(\mathrm{p}<0.05$, Fig. 3D). These results indicate that MCF-7 cells have very low metastatic potential. The wound closure ratios for the MDA-MB-231 cells were $68.00 \pm 4.16,86.00 \pm 9.89$ and $114.80 \pm 9.22$ in the control and 10 and $20 \%$ CM groups, respectively, and the values obtained for the CM groups were significantly higher compared with that obtained for the control group ( $\mathrm{p}<0.05$, Fig. 3D). 
A

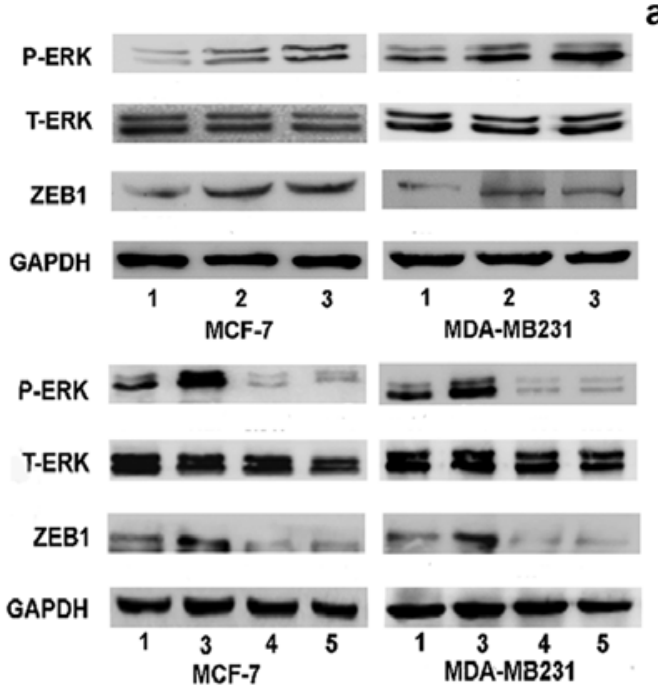

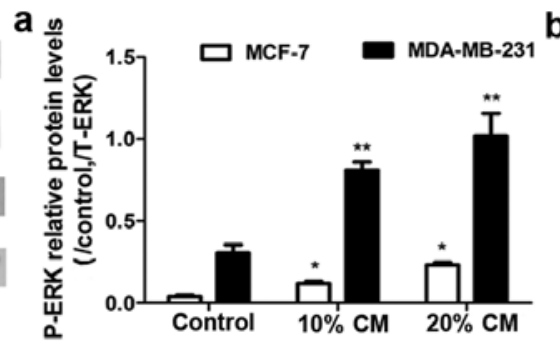

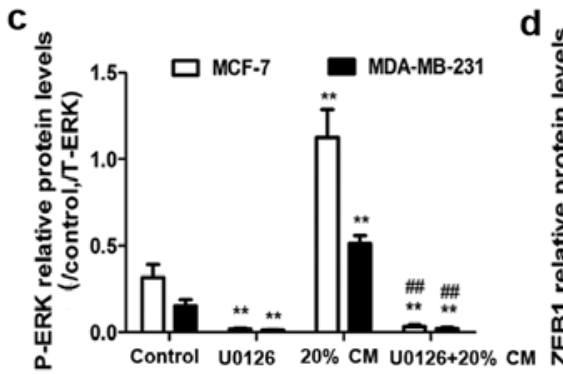

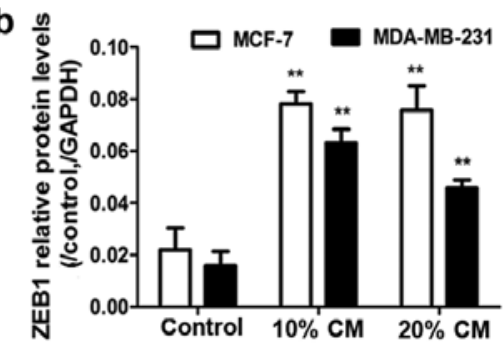

$\mathbf{d}_{\frac{0}{0}}$

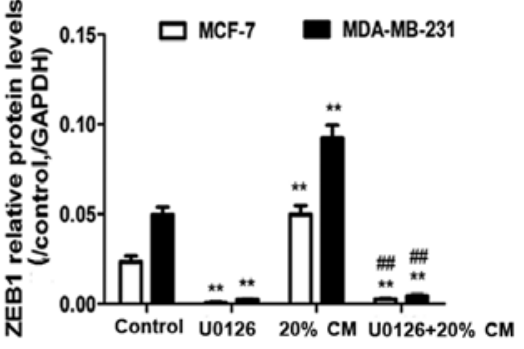

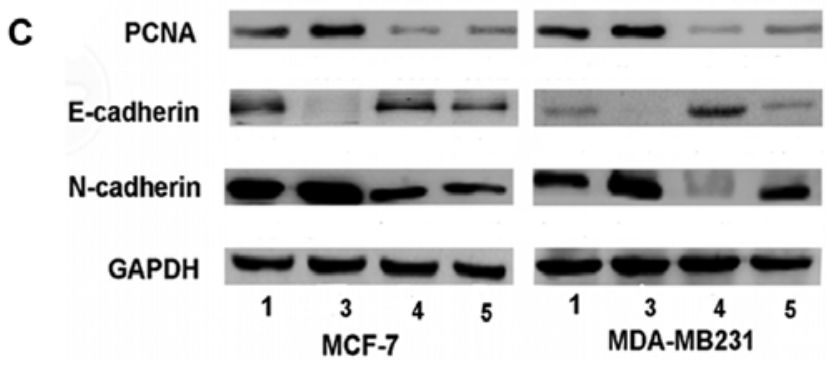
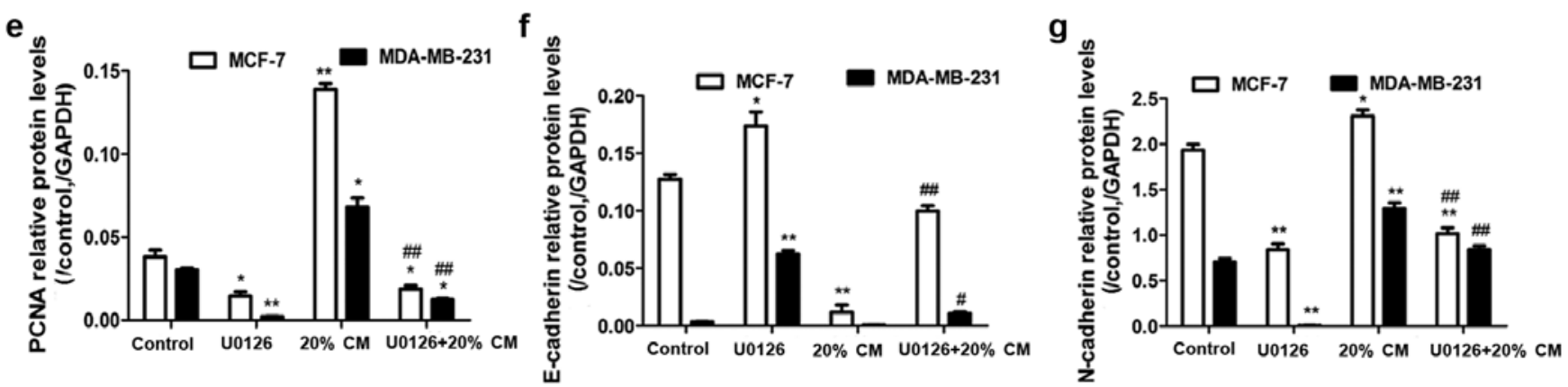

Figure 5. Effects of UC-MSCs and ERK inhibitor U0126 on the protein expression levels in the breast cancer cell lines. (A) MCF-7 and MDA-MB-231 cells were treated with 0,10 and 20\% UC-MSC-CM for $48 \mathrm{~h}$. The P-ERK and ZEB1 protein levels in MCF-7 and MDA-MB-231 cells were analyzed by western blotting. T-ERK and GAPDH protein levels were used as controls to ensure equal loading. Three independent experiments were performed to measure the (a) P-ERK and (b) ZEB1 protein levels. (B and C) MCF-7 and MDA-MB-231 cells were pretreated with $10 \mu \mathrm{M}$ U0126 for 60 min before the addition of 0 and $20 \%$ UC-MSC-CM. The P-ERK, ZEB1, E-cadherin, N-cadherin, and PCNA protein levels were analyzed by western blotting. Three independent experiments were used to measure the (c) P-ERK, (d) ZEB1, (e) PCNA, (f) E-cadherin and (g) N-cadherin protein levels. " $\mathrm{p}<0.05$ and ${ }^{* *}$ p $<0.01$ compared with the control group; ${ }^{\#} \mathrm{p}<0.05$ and ${ }^{\# \#} \mathrm{p}<0.01$ for the comparison between the U0126+20\% CM group and the $20 \% \mathrm{CM}$ group. Lane 1, control; lane 2, 10\% CM; lane 3, 20\% CM; lane 4, U0126; lane 5, U0126+20\% CM. CM, conditioned medium; UC-MSCs, umbilical cord mesenchymal stem cells.

E-cadherin and $N$-cadherin expression. The RT-PCR and western blot results showed that treatment with $\mathrm{CM}$ downregulated E-cadherin and increased N-cadherin (Fig. 4A and B, $\mathrm{p}<0.05)$. The results revealed that the mRNA and protein expression levels of $\mathrm{N}$-cadherin in breast cancer cells were significantly higher than those in the control group. In addition, there was a significant difference in the expression of E-cadherin in the MCF-7 and MDA-MB-231 cells between the $\mathrm{CM}$ and control groups. The $\mathrm{CM}$-induced migration of UC-MSCs may be achieved by the suppression of E-cadherin and the stimulation of $\mathrm{N}$-cadherin expression.
Protein expression of UC-MSCs and the effect of ERK inhibitor U0126 on breast cancer cells. To investigate whether $\mathrm{CM}$ downregulates E-cadherin expression by modulating the transcription factor ZEB1, we examined ZEB1 protein levels. Treatment with CM significantly increased ZEB1 expression by $~ 4$-fold in the MCF-7 cells and 3-fold in the MDA-MB-231 cells compared with the control groups ( $<<0.05$, Fig. 5Ab). These findings indicate that the effect of UC-MSCs on breast cancer cell migration may be achieved by EMT. We then analyzed the activation of the ERK pathway. Treatment with 10 and 20\% CM enhanced the p-ERK levels in the MCF-7 

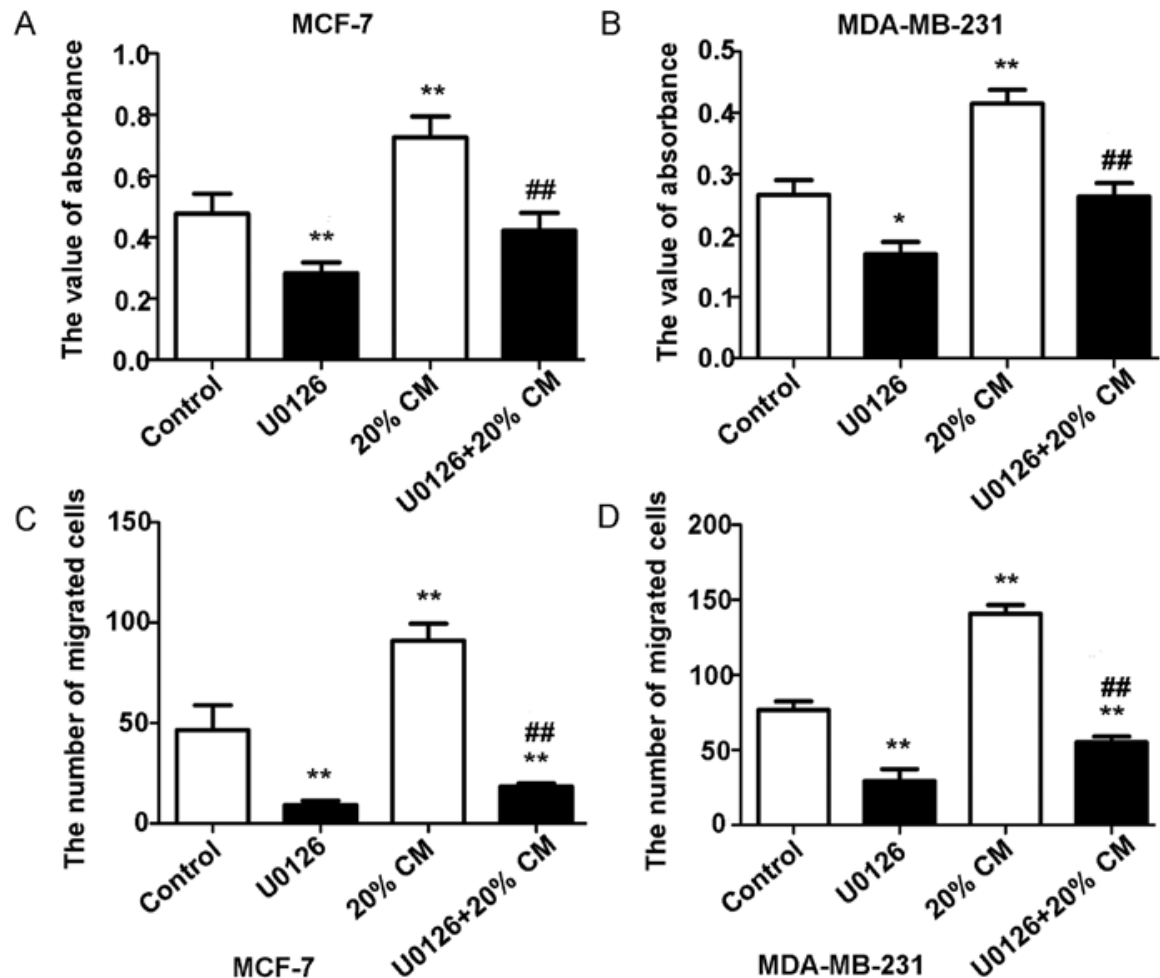

Figure 6. Effects of the ERK inhibitor U0126 on CM-stimulated cell proliferation and migration. After pretreatment with $10 \mu \mathrm{M} \mathrm{U} 0126$ for 60 min, MCF-7 and MDA-MB-231 cells were incubated with 0 and 20\% CM. MTT assay results for (A) MCF-7 and (B) for MDA-MB-231 cells. Transwell migration assay results for (C) MCF-7 and (D) for MDA-MB-231 cells in the absence or presence of U0126. " $\mathrm{p}<0.05$ and ${ }^{* *} \mathrm{p}<0.01$ compared with the control group; ${ }^{*} \mathrm{p}<0.05$ and ${ }^{\# \#} \mathrm{p}<0.01$ for the comparison of the U0126+20\% CM group with the $20 \%$ CM group.

and MDA-MB-231 cells ( $\mathrm{p}<0.05$, Fig. 5Aa). To determine whether the MAPK/ERK signaling pathway is involved in the CM-induced increase in ZEB1 protein levels, the cells were treated with the ERK inhibitor U0126 (Promega, Madison, WI, USA) in the presence or absence of CM. Notably, U0126 significantly decreased the ZEB1 and p-ERK protein levels (Fig. 5Bc and d). These results are consistent with those of previous studies that demonstrated that MAPK/ERK is an upstream factor of ZEB1 activation in ovarian cancer cells in vitro (24) and that MAPK/ERK signaling is required in IGF-1-induced ZEB1 expression in prostate cancer cells (25). The ERK inhibitors significantly decreased $\mathrm{N}$-cadherin expression and increased basal E-cadherin expression (Fig. 5Cf and g) as well as markedly diminished but not completely abolished the CM-induced suppression of E-cadherin expression. This finding suggests that MAPK/ERK signaling is required for MSC-derived CM-induced E-cadherin downregulation. Furthermore, U0126 significantly decreased the PCNA protein levels (Fig. 5Ce). Taken together, these results indicate that the MAPK/ERK pathway is involved in CM-induced breast cancer cell proliferation and migration.

Effects of the ERK inhibitor U0126 on CM-stimulated cell proliferation and migration. To determine whether the ERK pathway is involved in the effects of CM on breast cancer cells, we used the ERK inhibitor U0126 to specifically block the MAPK/ERK pathway in MCF-7 and MDA-MB-231 cells. As shown in Fig. 6A and B, the MTT assay results showed that U0126 treatment significantly decreased breast cancer cell proliferation $(\mathrm{p}<0.05)$. The proliferation rates obtained were
$0.477 \pm 0.065,0.2820 \pm 0.036,0.7267 \pm 0.068$ and $0.4220 \pm 0.058$ for MCF-7 cells and $0.267 \pm 0.048,0.170 \pm 0.040,0.415 \pm 0.045$, and $0.264 \pm 0.044$ for MDA-MB-231 cells. Our results also showed that CM-induced cell migration was markedly diminished but not totally abolished by treatment with U0126 ( $\mathrm{p}<0.05)$. The numbers of migrated MCF-7 cells were $46.50 \pm 12.40$, $9.200 \pm 2.168,91.00 \pm 8.524$ and $18.25 \pm 1.708$, whereas the numbers of migrated MDA-MB-231 cells were $78.33 \pm 4.509$, $28.67 \pm 2.082,138.7 \pm 7.572$ and $54.25 \pm 5.058$ (Fig. 6C and D). This finding also suggests that other pathways may be involved in the response of breast cancer cells to hUC-MSCs.

\section{Discussion}

In the present study, UC-MSCs from human umbilical cord tissues showed a homogenous immunophenotype and multilineage differentiation potential (osteoblast and adipocyte lineages). We demonstrated that these were homogeneously positive for the mesenchymal cell markers CD29, CD90 and CD44 but negative for CD34 and HLA-DR. These results are consistent with those of previous studies $(26,27)$. The data showed that UC-MSCs were Alizarin Red-positive and Oil Red O-positive after induction. Taken together, the findings suggest that the isolated adherent cells from the umbilical cord were in fact MSCs.

Furthermore, we observed the effects of UC-MSCs on the proliferation and migration of the human breast cancer cell lines MCF-7 and MDA-MB-231 in vitro. The MTT cell proliferation results showed that UC-MSC-CM significantly stimulates breast cancer cell proliferation. Therefore, it was 
suggested that UC-MSCs may exert certain increasing effects on the growth of breast cancer cells in vitro. The statistical analysis of the scratch wound and Transwell migration assay results revealed that $\mathrm{CM}$ significantly promoted $\mathrm{MCF}-7$ and MDA-MB-231 cell migration. Our results are consistent with those of previous studies (28-31).

E-cadherin functions as a cell-cell adhesion protein and tumor-suppressor that is silenced in many malignancies (32). E-cadherin is known to suppress tumor invasion, and the re-expression of E-cadherin in E-cadherin-deficient tumors reverts cells to a less invasive phenotype $(33,34)$. Some findings indicate that hMSCs decrease cell-to-cell contact and decrease epithelial cell adhesion markers (i.e., E-cadherin) in breast cancer cells $(35,36)$. Several transcription factors have been identified to suppress E-cadherin, including Twist, Snail, Slug and ZEB1, via their interaction with the E-box binding site in the E-cadherin promoter $(37,38)$. In the present study, we demonstrated that $\mathrm{CM}$ reduced the E-cadherin protein and mRNA levels and increased $\mathrm{N}$-cadherin and ZEB1 expression via activation of the MAPK/ERK signaling pathways. Finally, our results found that the downregulation of E-cadherin mediated by $\mathrm{CM}$ enhanced the migration of breast cancer cells.

PCNA is a well-defined regulator of DNA replication and cell cycle control (39). Treatment with CM significantly increased the PCNA protein levels. An inhibitor of ERK was able to downregulate the expression of PCNA. Furthermore, this effect was regulated through ERK nuclear translocation, resulting in enhanced PCNA expression. These findings suggest that UC-MSC-CM induced the proliferation of MCF-7 and MDA-MB-231 cells via the MAPK/ERK pathways.

However, our results are contrary to those of previous studies, which suggest that hUC-MSCs inhibit the growth of breast cancer cells $(40,41)$. We speculate that this discrepancy may be related to the sources and numbers of the MSCs, differences in the culture and experimental methods, the type and site of the carcinoma, or a combination of these factors. We believe that UC-MSCs provide potential for cancer therapy, and further study of UC-MSCs will offer a better understanding of the relationship between MSCs and tumor progression and the mechanism governing this relationship.

\section{Acknowledgements}

The present study was supported by the Foundation of Jiangsu University for Seniors (grant no. 11JDG0089) and the Innovation Project of Cultivating Graduates of Jiangsu Province (grant no. CXLX13_689) and the Science Foundation of Kunshan (grant no. KS1331).

\section{References}

1. Nelson HD, Zakher B, Cantor A, Fu R, Griffin J, O'Meara ES, Buist DS, Kerlikowske K, van Ravesteyn NT, Trentham-Dietz A, et al: Risk factors for breast cancer for women aged 40 to 49 years: A systematic review and meta-analysis. Ann Intern Med 156: 635-648, 2012.

2. Jemal A, Bray F, Center MM, Ferlay J, Ward E and Forman D: Global cancer statistics. CA Cancer J Clin 61: 69-90, 2011.

3. Hugosson J, Stranne J and Carlsson SV: Radical retropubic prostatectomy: A review of outcomes and side-effects. Acta Oncol 50 (Suppl 1): 92-97, 2011.
4. De Ruysscher D, Van Meerbeeck J, Vandecasteele K, Oberije C, Pijls M, Dingemans AM, Reymen B, van Baardwijk A, Wanders R, Lammering G, et al: Radiation-induced oesophagitis in lung cancer patients. Is susceptibility for neutropenia a risk factor? Strahlenther Onkol 188: 564-567, 2012.

5. Mbeunkui F and Johann DJ Jr: Cancer and the tumor microenvironment: A review of an essential relationship. Cancer Chemother Pharmacol 63: 571-582, 2009.

6. Orimo A, GuptaPB,Sgroi DC,Arenzana-Seisdedos F,Delaunay T, Naeem R, Carey VJ, Richardson AL and Weinberg RA: Stromal fibroblasts present in invasive human breast carcinomas promote tumor growth and angiogenesis through elevated SDF-1/CXCL12 secretion. Cell 121: 335-348, 2005

7. Karnoub AE, Dash AB, Vo AP, Sullivan A, Brooks MW, Bell GW, Richardson AL, Polyak K, Tubo R and Weinberg RA: Mesenchymal stem cells within tumour stroma promote breast cancer metastasis. Nature 449: 557-563, 2007.

8. Whiteside TL: The tumor microenvironment and its role in promoting tumor growth. Oncogene 27: 5904-5912, 2008.

9. Shinagawa K, Kitadai Y, Tanaka M, Sumida T, Kodama M, Higashi Y, Tanaka S, Yasui W and Chayama K: Mesenchymal stem cells enhance growth and metastasis of colon cancer. Int J Cancer 127: 2323-2333, 2010.

10. Yu JM, Jun ES, Bae YC and Jung JS: Mesenchymal stem cells derived from human adipose tissues favor tumor cell growth in vivo. Stem Cells Dev 17: 463-473, 2008.

11. Khakoo AY, Pati S, Anderson SA, Reid W, Elshal MF, Rovira II, Nguyen AT, Malide D, Combs CA, Hall G, et al: Human mesenchymal stem cells exert potent antitumorigenic effects in a model of Kaposi's sarcoma. J Exp Med 203: 1235-1247, 2006.

12. Lee JK, Park SR, Jung BK, Jeon YK, Lee YS, Kim MK, Kim YG, Jang JY and Kim CW: Exosomes derived from mesenchymal stem cells suppress angiogenesis by down-regulating VEGF expression in breast cancer cells. PLoS One 8: e84256, 2013.

13. Gauthaman K, Yee FC, Cheyyatraivendran S, Biswas A, Choolani M and Bongso A: Human umbilical cord Wharton's jelly stem cell (hWJSC) extracts inhibit cancer cell growth in vitro. J Cell Biochem 113: 2027-2039, 2012.

14. Rao MS and Mattson MP: Stem cells and aging: Expanding the possibilities. Mech Ageing Dev 122: 713-734, 2001.

15. Secco M, Zucconi E, Vieira NM, Fogaça LL, Cerqueira A, Carvalho MD, Jazedje T, Okamoto OK, Muotri AR and Zatz M: Multipotent stem cells from umbilical cord: Cord is richer than blood! Stem Cells 26: 146-150, 2008.

16. Baksh D, Yao R and Tuan RS: Comparison of proliferative and multilineage differentiation potential of human mesenchymal stem cells derived from umbilical cord and bone marrow. Stem Cells 25: 1384-1392, 2007.

17. Ayuzawa R, Doi C, Rachakatla RS, Pyle MM, Maurya DK, Troyer D and Tamura M: Naïve human umbilical cord matrix derived stem cells significantly attenuate growth of human breast cancer cells in vitro and in vivo. Cancer Lett 280: 31-37, 2009.

18. Xu WT, Bian ZY, Fan QM, Li G and Tang TT: Human mesenchymal stem cells (hMSCs) target osteosarcoma and promote its growth and pulmonary metastasis. Cancer Lett 281: 32-41, 2009.

19. Rachakatla RS, Pyle MM, Ayuzawa R, Edwards SM, Marini FC, Weiss ML, Tamura M and Troyer D: Combination treatment of human umbilical cord matrix stem cell-based interferon-beta gene therapy and 5-fluorouracil significantly reduces growth of metastatic human breast cancer in SCID mouse lungs. Cancer Invest 26: 662-670, 2008.

20. Ganta C, Chiyo D, Ayuzawa R, Rachakatla R, Pyle M, Andrews G, Weiss M, Tamura M and Troyer D: Rat umbilical cord stem cells completely abolish rat mammary carcinomas with no evidence of metastasis or recurrence 100 days post-tumor cell inoculation. Cancer Res 69: 1815-1820, 2009.

21. Moodley Y, Atienza D, Manuelpillai U, Samuel CS, Tchongue J, Ilancheran S, Boyd R and Trounson A: Human umbilical cord mesenchymal stem cells reduce fibrosis of bleomycin-induced lung injury. Am J Pathol 175: 303-313, 2009.

22. Caldas-Lopes E, Cerchietti L, Ahn JH, Clement CC, Robles AI, Rodina A, Moulick K, Taldone T, Gozman A, Guo Y, et al: Hsp90 inhibitor PU-H71, a multimodal inhibitor of malignancy, induces complete responses in triple-negative breast cancer models. Proc Natl Acad Sci USA 106: 8368-8373, 2009.

23. Soule HD, Vazguez J, Long A, Albert S and Brennan M: A human cell line from a pleural effusion derived from a breast carcinoma. J Natl Cancer Inst 51: 1409-1416, 1973. 
24. Lau MT, So WK and Leung PC: Fibroblast growth factor 2 induces E-cadherin down-regulation via PI3K/Akt/mTOR and MAPK/ERK signaling in ovarian cancer cells. PLoS One 8: e59083, 2013.

25. Graham TR, Zhau HE, Odero-Marah VA, Osunkoya AO, Kimbro KS, Tighiouart M, Liu T, Simons JW and O'Regan RM: Insulin-like growth factor-I-dependent up-regulation of ZEB1 drives epithelial-to-mesenchymal transition in human prostate cancer cells. Cancer Res 68: 2479-2488, 2008.

26. Qiao C, Xu W, Zhu W, Hu J, Qian H, Yin Q, Jiang R, Yan Y, Mao $\mathrm{F}$ and Yang H: Human mesenchymal stem cells isolated from the umbilical cord. Cell Biol Int 32: 8-15, 2008.

27. Fong CY, Richards M, Manasi N, Biswas A and Bongso A: Comparative growth behaviour and characterization of stem cells from human Wharton's jelly. Reprod Biomed Online 15: 708-718, 2007.

28. Zhu W, Huang L, Li Y, Qian H, Shan X, Yan Y, Mao F, Wu X and Xu WR: Mesenchymal stem cell-secreted soluble signaling molecules potentiate tumor growth. Cell Cycle 10: 3198-3207, 2011.

29. Zhang T, Lee YW, Rui YF, Cheng TY, Jiang XH and Li G: Bone marrow-derived mesenchymal stem cells promote growth and angiogenesis of breast and prostate tumors. Stem Cell Res Ther 4: 70, 2013.

30. Ke CC, Liu RS, Suetsugu A, Kimura H, Ho JH, Lee OK and Hoffman RM: In vivo fluorescence imaging reveals the promotion of mammary tumorigenesis by mesenchymal stromal cells. PLoS One 8: e69658, 2013.

31. Yan XL, Fu CJ, Chen L, Qin JH, Zeng Q, Yuan HF, Nan X, Chen HX, Zhou JN, Lin YL, et al: Mesenchymal stem cells from primary breast cancer tissue promote cancer proliferation and enhance mammosphere formation partially via EGF/EGFR/Akt pathway. Breast Cancer Res Treat 132: 153-164, 2012.

32. Nollet F, Berx G and van Roy F: The role of the E-cadherin/catenin adhesion complex in the development and progression of cancer. Mol Cell Biol Res Commun 2: 77-85, 1999.
33. Gottardi CJ, Wong E and Gumbiner BM: E-cadherin suppresses cellular transformation by inhibiting beta-catenin signaling in an adhesion-independent manner. J Cell Biol 153: 1049-1060, 2001.

34. Yanagisawa M and Anastasiadis PZ: p120 catenin is essential for mesenchymal cadherin-mediated regulation of cell motility and invasiveness. J Cell Biol 174: 1087-1096, 2006.

35. Fierro FA, Sierralta WD, Epuñan MJ and Minguell JJ: Marrow-derived mesenchymal stem cells: Role in epithelial tumor cell determination. Clin Exp Metastasis 21: 313-319, 2004.

36. Martin FT, Dwyer RM, Kelly J, Khan S, Murphy JM, Curran C, Miller N, Hennessy E, Dockery P, Barry FP, et al: Potential role of mesenchymal stem cells (MSCs) in the breast tumour microenvironment: Stimulation of epithelial to mesenchymal transition (EMT). Breast Cancer Res Treat 124: 317-326, 2010.

37. Bolós V, Peinado H, Pérez-Moreno MA, Fraga MF, Esteller M and Cano A: The transcription factor Slug represses E-cadherin expression and induces epithelial to mesenchymal transitions: A comparison with Snail and E47 repressors. J Cell Sci 116: 499-511, 2003.

38. Peinado H, Olmeda D and Cano A: Snail, Zeb and bHLH factors in tumour progression: An alliance against the epithelial phenotype? Nat Rev Cancer 7: 415-428, 2007.

39. Strzalka W and Ziemienowicz A: Proliferating cell nuclear antigen (PCNA): A key factor in DNA replication and cell cycle regulation. Ann Bot (Lond) 107: 1127-1140, 2011.

40. Sun B, Yu KR, Bhandari DR, Jung JW, Kang SK and Kang KS: Human umbilical cord blood mesenchymal stem cell-derived extracellular matrix prohibits metastatic cancer cell MDA-MB-231 proliferation. Cancer Lett 296: 178-185, 2010.

41. Ma Y, Hao X, Zhang S and Zhang J: The in vitro and in vivo effects of human umbilical cord mesenchymal stem cells on the growth of breast cancer cells. Breast Cancer Res Treat 133: 473-485, 2012. 